\title{
Bourse SFRP
}

La SFRP a attribué une bourse à ces jeunes étudiants pour leur stage de Master $2^{e}$ année de recherche. Nous avons le plaisir de publier dans cette rubrique destinée aux jeunes chercheurs un résumé de leur stage. Nous leur souhaitons de continuer brillamment leur carrière en souhaitant qu'ils restent attachés à la radioprotection.

\section{Implication d'ATM dans la réponse aux radiations de la chorée de Huntington}

Stage réalisé à l'INSERM CR-U1052, Centre de recherche en cancérologie de Lyon (Directeur : Alain PUISIEUX) sous la responsabilité de Nicolas FORAY.

La maladie de Huntington (MH) est un syndrome neurodégénératif causé par une mutation sur le gène IT15 codant pour la huntingtine (Htt). Peu de données sont disponibles à propos du rôle biologique de la Htt, notamment concernant la réponse au stress génotoxique. Cependant, dans les années 80, quelques groupes de recherche ont montré que la MH était associée à une certaine radiosensibilité. Toutefois, aucun modèle n'a été proposé pour expliquer une telle caractéristique. Dans le cadre de ce stage de M2, la réponse radioinduite de différentes protéines impliquées dans le processus de réparation des cassures double-brin (CDB) a été systématiquement étudiée. Après une irradiation de 2 Gy de rayons gamma, deux lignées de fibroblastes cutanés provenant de patients $\mathrm{MH}$ ont montré un défaut de reconnaissance des CDB reflétés par un faible taux de foci $\mathrm{pH} 2 \mathrm{AX}$ détecté par immunofluorescence. Ces deux lignées MH montrent aussi un retard dans le transit nucléocytoplasmique de la protéine ATM et/ou un défaut de son activité kinase. Une combinaison de biphosphonate et de statine (ZOPRA) corrige ces défauts.

Jusqu'à présent, l'une des approches majeures de la radioprotection chimique était de contribuer à diminuer le stress oxydatif pour diminuer le nombre de dommages de l'ADN (ex thiols). À la lumière de nos résultats, la combinaison ZOPRA apparaitrait comme un stimulateur de la réparation par augmentation du transit de pATM du cytoplasme vers le noyau. Cette approche diffère clairement des stratégies de radioprotection utilisées jusqu'à présent. Par exemple, les thiols peuvent diminuer le nombre de cassures induites mais ne diminuent pas le nombre de cassures non réparées. Ainsi en se basant sur les données obtenues dans le laboratoire sur des syndromes comme la Progéria (Varela et al., 2008) la Neurofibromatose, la tubéreuse de Bourneville et la maladie de Bruton, on peut imaginer l'application du ZOPRA comme radioprotecteur efficace tout en espérant un effet bénéfique sur le tableau clinique de ces maladies.

Varela I., Pereira S., Ugalde A.P., Navarro C.L., Suárez M.F., Cau P., Cadiñanos J., Osorio F.G., Foray N., Cobo J., de Carlos F., Lévy N., Freije J.M., López-Otín C. (2008) Combined treatment with statins and aminobisphosphonates extends longevity in a mouse model of human premature aging, Nat. Med. 14 (7), 767-772.

Mélanie FERLAZZO 